\title{
COMPORTAMENTO ALIMENTAR DE POTROS DA RAÇA MANGALARGA MARCHADOR SUBMETIDOS A OFERTAS DE ALIMENTO E CONFINAMENTO NOTURNO
}

\author{
João Ricardo Dittrich ${ }^{1}$, Adir de Sá Neto ${ }^{1}$, Dayana Swaroski ${ }^{1}$, Alex Henrique Lobo ${ }^{1}$, \\ Fabiola Cassanelli ${ }^{1}$, Helen Aline Melo ${ }^{1}$ \\ 1 Universidade Federal do Paraná - dittrich@ufpr.br
}

\begin{abstract}
RESUMO: Objetivou-se neste experimento avaliar o comportamento alimentar de potros da raça Mangalarga Marchador, submetidos a dietas em cocheira e em pastagem de Hemarthria altíssima cv. Flórida, no período de 29/09/2007 à 29/02/2008. O experimento foi realizado no Haras Jump localizado na região metropolitana de Curitiba. Os tratamentos utilizados foram dietas compostas de $5 \mathrm{~kg}$ feno e 2,5 $\mathrm{Kg}$ de concentrado comercial oferecido na cocheira durante noite e pastejo durante o dia (T100C), $2,5 \mathrm{Kg}$ feno e $1,250 \mathrm{Kg}$ de concentrado comercial oferecido na cocheira durante a noite e pastejo durante o dia (T50C), e somente alimento na forma de pasto (TP). O comportamento alimentar foi monitorado nos dias 07/12/2007, 30/01/2008 e 19/02/2008, quantificando-se o tempo de alimentação durante 24 horas, por meio de amostragem focal, a cada 10 minutos. Os resultados foram submetidos à análise de variância e as medias comparadas pelo teste de Duncan, pelo programa Statistica versão 5.5. Os animais do tratamento TP apresentaram maior tempo de ingestão de alimento no dia. O comportamento de pastejo dos potros, alimentados na cocheira a noite, foi influenciado pelo confinamento, o que determinou menor $(P<0,05)$ tempo de ingestão no período da manhã, assim como o incremento no período da tarde, relativo à manhã. A velocidade de colheita da forragem, no período diurno, foi maior $(\mathrm{P}<0,05)$ para os animais do tratamento $T 50 \mathrm{C}$ e $T 100 \mathrm{C}$. $\mathrm{O}$ peso e escore corporal dos potros no período experimental foram satisfatórios para os três tratamentos. O confinamento de potros no período noturno, suplementados com concentrado e feno a vontade, promove diminuição do tempo de ingestão alimentar diário.
\end{abstract}

Palavras-chave: dietas; hemartria; tempo de pastejo

\section{FEEDING BEHAVIOR OF MANGALARGA MARCHADOR FOALS UNDER FOOD OFFERS AND NOCTURNE STABLED}

\begin{abstract}
The aim of this study was identifies the feeding behavior of Mangalarga Marchador foals on the stable at night and full time at the limpograss (Hemarthria altissima cv. Flórida). This experiment took place in Haras Jump ,Curitiba - PR, from 09/29/07 to 02/28/08. The treatments were diets compound by haygrass $(5,0 \mathrm{~kg})$ and commercial concentrate $(2,5 \mathrm{~kg})$ offered on the stable during night and at the pasture during daylight $(\mathrm{T} 100 \mathrm{C})$, haygrass $(2,5 \mathrm{~kg})$ and commercial concentrate $(1,25 \mathrm{~kg})$ offered on the stable during night and at the pasture during daylight (T50C), and only limpograss pasture (TP). The feeding behavior was measured by focal animal sampling, each 10 minutes, on 12/07/2007, 01/30/2008 and 02/19/2008, during 24 hours. The development was control by weight gain and body score. The results were analyzed by Statistica, version 5.5, program. The foals of TP treatments (only limpograss) feeding how long during day that nocturne stabled foals. The stable influenced the foals feeding behavior at the pasture, because the time feeding morning was smaller $(P<0.05)$ than afternoon and TP treatments. The speed of grazing during daylight was higher $(P<0.05)$ than stabled treatments. The weight and body score of the foals were satisfactory. Foals on nocturne housing decrease the ingestion daily time even with concentrate and hay $a d$ libitum supply.
\end{abstract}

Key Words: diet; limpograss; grazing time 


\section{INTRODUÇÃO}

Os conceitos de alimentação e nutrição de eqüinos, de uma forma objetiva, indicam as quantidades diárias necessárias de classes de alimentos, nas quais estão incluídos os concentrados e volumosos (NRC, 2007). Considerando estes conceitos, adota-se na maioria dos centros de criação de eqüinos, o modelo alimentar que prioriza a ingestão de nutrientes em alimentos concentrados, tais como rações comerciais, produtos e coprodutos da agroindústria, devido à facilidade na aquisição e a disponibilidade desse tipo de alimento com bom valor nutritivo. Os alimentos volumosos, na maioria das vezes, não recebem a atenção devida tanto em relação às características quantitativas quanto qualitativas. Apesar da comprovada necessidade da ingestão diária de volumosos com bom valor nutricional, a forma como este alimento é disponibilizado aos cavalos não tem sido foco principal das pesquisas. Estas têm apresentado informações limitadas a respeito das interações comportamentais dos cavalos frente às diferentes formas como pastos, capineiras, fenos, silagens e pré-secados (NRC, 2007). A carência de informações científicas tem levado à adoção de práticas de manejo que desconsideram a quantidade, a qualidade e a forma como os cavalos selecionam e colhem este alimento (Dittrich et al., 2005), o que pode acarretar em inadequada ingestão de nutrientes.

A prática de manejo adotada na maioria das fazendas de criação e centros de treinamento é a utilização de forragens conservadas na forma de fenos de gramíneas e leguminosas, disponibilizados em cocheiras ou mesmo nas áreas de pastagem. Este manejo se faz necessário principalmente devido a restrição do tamanho das áreas disponíveis para o pastejo, do tempo disponibilizado para o cavalo se alimentar no dia, da diversidade de alternativas alimentares e do manejo inadequado das pastagens (Singer, 1999).

O pasto constitui-se na principal fonte alimentar para os eqüinos devido às características evolutivas desta espécie (Janis, 1975), quando disponíveis em quantidade, qualidade e diversidade, permitem aos animais a seletividade, a qual é a principal ferramenta de qualquer herbívoro para melhorar a qualidade da dieta (Dittrich et al., 2007). Outro fator de impacto é o tempo disponível para o pastejo, o qual na equinocultura é constantemente influenciado pelo confinamento dos animais, principalmente no período noturno. A diminuição do tempo disponível para o cavalo colher seu alimento determina alterações importantes tanto no comportamento alimentar quanto no próprio bem estar, comprovado pela frequente ocorrência de estereotipias em animais estabulados (Johnson et al., 1998).

A relação de fatores como tipo do alimento disponível sua quantidade e qualidade, bem como o tempo disponível aos animais para se alimentar são de extrema importância na identificação das melhores estratégias de manejo a serem praticadas, tanto em fazendas de criação quanto em centros de treinamento. Objetivou-se neste experimento avaliar como o confinamento noturno e a oferta de alimentos volumosos e concentrados, fatores de manejo dos animais, influenciam os aspectos do comportamento alimentar de potros em crescimento.

\section{MATERIAL E MÉTODOS}

O experimento foi realizado no Haras Jump, localizado no município de 
Piraquara, região metropolitana de Curitiba, estado do Paraná, situado a 900m de altitude. A região possui clima Cfb, segundo a classificação de Köeppen, com temperatura média anual entre 16 e $1^{\circ} \mathrm{C}$, precipitação média anual próxima de $2000 \mathrm{~mm}$ e umidade relativa do ar acima de $80 \%$ (IAPAR, 2006). O período experimental foi de $15 / 10 / 07$ à $29 / 02 / 08$. Foram utilizados 12 potros da raça Mangalarga Marchador com, aproximadamente, 12 meses de idade e peso corporal médio de 187 quilos, distribuídos em um delineamento experimental inteiramente casualizado, sendo que para cada tratamento foram utilizados quatro animais, separados em dupla de sexos diferentes.

Três foram os tratamentos utilizados, sendo dois em confinamento noturno e outro exclusivamente em pastagem. Para os confinados foram disponibilizadas, durante a noite, dietas baseadas em 100\% (T100C) e 50\% (T50C) das quantidades necessárias em matéria seca (MS) no dia, estabelecidas pelo NRC (2007). Para o tratamento exclusivamente em pastagem (TP), os animais permaneceram 24 horas por dia em pastagem de Hemarthria altíssima cv. Flórida (TP). Para tanto, no tratamento T100C foi utilizado $5 \mathrm{~kg}$ feno de azevém (Lolium multiflorum) e $2,5 \mathrm{~kg}$ de concentrado comercial oferecidos na cocheira durante o período noturno e pastejo durante o diurno. No tratamento T50C, $2,5 \mathrm{~kg}$ feno e 1,25 kg de concentrado comercial foram oferecidos na cocheira durante o período noturno e pastejo durante o diurno e no tratamento TP somente alimento na forma de pasto nos períodos noturno e diurno. Os animais do tratamento T100C e T50C durante 0 período diurno foram mantidos em piquetes de Hemartria, com área de 0,6 ha e oferta de $8 \%$, ajustada pela técnica "put and take" (Mott e Lucas, 1952), o mesmo ajuste de oferta foi utilizado para os animais do tratamento TP. Em todos os piquetes disponibilizou-se água e sal mineral ad libitum.

Para controle do desenvolvimento corporal os animais foram pesados, após quatro horas de jejum alimentar e hídrico, em balança eletrônica da marca Toledo, de capacidade até $500 \mathrm{~kg}$ e submetidos a avaliação do escore corporal por meio da escala de um a nove (Henneke et al., 1983). As pesagens e avaliações dos escores corporais foram nos dias 01/11/07, 07/12/07, 30/01/08 e 19/02/08.

O concentrado foi disponibilizado aos animais dos tratamentos T100C e T50C as 8:00 horas e as 18:00 horas, o feno apenas no horário da tarde. Após a ingestão do concentrado no período da manhã os animais eram transferidos para os piquetes de Hemártria. $O$ feno não consumido (sobras) no período noturno era recolhido e pesado diariamente. A composição bromatológica do feno, do concentrado e do pasto está apresentada na Tabela 1.

Os animais foram adaptados ao ambiente experimental e aos tratamentos por sete dias, após esse período o comportamento ingestivo foi identificado pela avaliação dos animais durante 24 horas nos dias 7 de dezembro, 30 de janeiro e 19 de fevereiro. O monitoramento era iniciado as 19:00 horas e terminava as 19:00 horas do dia seguinte. As avaliações foram divididas em noite (20:00 horas até 1:00 hora), madrugada (1:10 hora até 6:00horas), manhã (6:10 horas até 13:00 horas) e tarde (13:10 horas até 19:50 horas), levando-se em consideração as horas com luminosidade natural. As avaliações foram caracterizadas pelo monitoramento individual dos animais experimentais, a cada 10 minutos, de forma pontual, direta e contínua (Martin e Bateson, 1986), sendo que o comportamento de pastejo ou não pastejo, tanto para o pasto quanto para 
Tabela 1 - Composição bromatológica (100\% M.S.) do concentrado, do feno e da forragem colhida por simulação do pastejo em Proteína Bruta (PB), Extrato Etéreo (EE), Fibra em Detergente Ácido (FDA), Fibra em Detergente Neutro (FDN) e Resíduo Mineral (RM) e Energia Digestível (E.D.)

\begin{tabular}{lcccccc}
\multicolumn{1}{c}{ Alimento } & $\begin{array}{l}\text { PB } \\
(\%)\end{array}$ & $\begin{array}{l}\text { EE } \\
(\%)\end{array}$ & $\begin{array}{c}\text { FDA } \\
(\%)\end{array}$ & $\begin{array}{c}\text { FDN } \\
(\%)\end{array}$ & $\begin{array}{c}\text { RM } \\
(\%)\end{array}$ & $\begin{array}{c}\text { E.D. }{ }^{1} \\
(\mathrm{Mcal} / \mathrm{kg})\end{array}$ \\
\hline $\begin{array}{l}\text { Concentrado } \\
\begin{array}{l}\text { multiflorum) } \\
\text { Hemarthria altissima cv. }\end{array}\end{array}$ & 20,5 & 2,2 & 4,72 & 5,2 & 11,4 & 3,81 \\
Flórida & 11,7 & 2,6 & 43,4 & 72,2 & 10,6 & 2,66 \\
\hline
\end{tabular}

${ }^{1}$ Energia Digestível (Mcal/kg) calculada de acordo com NRC, 2007.

o feno, foi registrado por meio de amostragem focal e anotado em uma folha própria de avaliação. A soma desse tempo total diário caracterizou tempo de ingestão de feno e pastagem dos tratamentos.

Os animais dos tratamentos T100C e T50C foram recolhidos diariamente ao confinamento no início da noite, onde encontravam disponível o alimento concentrado e o feno nas citadas quantidades. $\mathrm{O}$ monitoramento da ingestão do feno era iniciado após a ingestão do alimento concentrado, conforme método descrito acima.

A velocidade de colheita da pastagem pelos animais, no período diurno, foi avaliada por meio da técnica de quantificação do tempo necessário para a formação de 20 bocados, com auxílio de cronômetro e contador (Hodgson, 1982), caracterizando, assim, a taxa de bocados demonstrada como bocados por minuto. A taxa de bocados, avaliada no período diurno, associada ao tempo de pastejo diurno permitiu estimar a velocidade de colheita do pasto no período.

Os resultados foram submetidos à análise de variância $e$ as médias comparadas pelo teste de Duncan, pelo programa Statistica, versão 5.5 .

\section{RESULTADOS E DISCUSSÃO}

Os animais do tratamento TP (só pastagem) destinaram maior $(P<0,05)$ tempo diário à ingestão de alimento, comparativamente aos tratamentos
T50C e T100C. O tempo diário de pastejo no tratamento TP está próximo aos apresentados por vários autores que estudaram 0 comportamento ingestivo de equinos em pastagens (Duncan, 1980; Winskill et al., 1996, Dittrich, 2001; Zanine et al., 2006). Entre os períodos do dia, o tempo de alimentação dos animais do tratamento TP foi superior $(P<0,05)$ durante a noite, em relação ao de madrugada e de manhã. $O$ confinamento e $o$ fornecimento de concentrado e feno no período noturno, em $50 \%$ e $100 \%$ das quantidades necessárias de alimento no dia (T50c e T100C), promoveram a diminuição do tempo de alimentação nos períodos da noite, madrugada e manhã (Tabela 2). No período da tarde houve similaridade do tempo de alimentação dos animais entre os tratamentos. Trabalhos que avaliaram o confinamento de éguas por 12 horas, tanto no período diurno quanto noturno, mostraram que o confinamento interfere no comportamento ingestivo quando comparadas com aquelas que ficam soltas 24 horas. Éguas soltas durante 24 horas pastejam proporcionalmente mais tempo do que aquelas presas durante 12 horas (Pond, 2000).

$\mathrm{Da}$ quantidade total de feno disponibilizado para os animais confinados durante a noite sobrou em média $49 \%$ para o tratamento $\mathrm{T} 100 \mathrm{C}$ e $53 \%$ para o T50C $(P>0,05)$. A quantidade de concentrado disponibilizada aos animais não influenciou a quantidade de feno consumida e o 
Tabela 2 - Tempo diário (horas e minutos) de ingestão de alimento entre os tratamentos

\begin{tabular}{|c|c|c|c|c|c|}
\hline Tratamento & Noite & Madrugada & Manhã & Tarde & Total \\
\hline $\mathrm{TP}$ & $2 \mathrm{~h}: 45^{\prime a}$ & $2 \mathrm{~h}: 12^{\prime} \mathrm{a}$ & $5 \mathrm{~h}: 43^{\prime}$ aA & $5 \mathrm{~h}: 30^{\prime}{ }^{\mathrm{AA}}$ & 16h:11,a \\
\hline T50C & $1 \mathrm{~h}: 38^{\prime} \mathrm{b}$ & Oh:40, b & $4 h: 26^{\prime}$ bA & $5 \mathrm{~h}: 33^{\prime}{ }^{\mathrm{aB}}$ & $12 \mathrm{~h}: 18^{\prime}$ \\
\hline T100C & $1 \mathrm{~h}: 53^{\prime} \mathrm{b}$ & Oh:49, b & $4 h: 14^{\text {, bA }}$ & $5 \mathrm{~h}: 18{ }^{\prime a B}$ & $12 \mathrm{~h}: 15^{, b}$ \\
\hline
\end{tabular}

Letras diferentes, minúsculas na coluna e maiúsculas na linha diferem entre si pelo teste de Duncan $(P<0,05)$.

Tabela 3 - Tempo de pastejo, taxa de bocados e número de bocados no período diurno (taxa de bocados $\mathrm{x}$ tempo de pastejo)

\begin{tabular}{|c|c|c|c|c|c|c|}
\hline Tratamento & Períodos & $\begin{array}{l}\text { Tempo de } \\
\text { pastejo } \\
\text { (horas) }\end{array}$ & $\begin{array}{l}\text { Bocados } \\
\text { por minuto }\end{array}$ & $\begin{array}{l}\text { Bocados por } \\
\text { minuto entre } \\
\text { períodos }\end{array}$ & $\begin{array}{l}\text { Bocados } \\
\text { totais por } \\
\text { período }\end{array}$ & $\begin{array}{l}\text { Bocados no } \\
\text { período diurno }\end{array}$ \\
\hline TP & $\begin{array}{l}\text { MNH } \\
\text { TDE }\end{array}$ & $\begin{array}{l}\text { 5h:43' a } \\
5 \mathrm{~h}: 30 \text { ' a }\end{array}$ & $25,12^{b}$ & $\begin{array}{l}24,69 \mathrm{a} \\
25,44 \mathrm{a}\end{array}$ & $\begin{array}{l}8.468^{\mathrm{b}} \\
8.395^{\mathrm{b}}\end{array}$ & 16.863 \\
\hline T50C & $\begin{array}{l}\text { MNH } \\
\text { TDE }\end{array}$ & $\begin{array}{l}\text { 4h:26' b } \\
5 \mathrm{~h}: 33 \text { ' a }\end{array}$ & $27,02^{a}$ & $\begin{array}{l}25,92 \mathrm{a} \\
27,94 \mathrm{~b}\end{array}$ & $\begin{array}{l}6.894^{\mathrm{c}} \\
9.304^{\mathrm{a}}\end{array}$ & 16.198 \\
\hline T100C & $\begin{array}{l}\text { MNH } \\
\text { TDE }\end{array}$ & $\begin{array}{l}\text { 4h:14' b } \\
5 \mathrm{~h}: 18^{\prime} \mathrm{a}\end{array}$ & $26,82^{a}$ & $\begin{array}{l}25,70 \mathrm{a} \\
28,27 \mathrm{~b}\end{array}$ & $\begin{array}{c}7.180^{c} \\
8.172^{b, c}\end{array}$ & 15.352 \\
\hline
\end{tabular}

Letras minúsculas diferentes na coluna diferem entre si pelo teste de Duncan $(\mathrm{P}<0,05)$.

tempo de consumo deste alimento. Os animais do tratamento $\mathrm{T} 50 \mathrm{C}$, os quais receberam menores quantidades de concentrado não incrementaram o consumo de feno, tanto em tempo quanto em quantidade (Tabela 2). Este comportamento provavelmente está relacionado à concentração de glicose plasmática, após a ingestão de concentrados, a qual é um dos fatores endógenos que controlam o consumo (Ralston, 1982). O confinamento no período noturno associado à ingestão de concentrados é uma prática alimentar que prejudica o bem estar dos animais por aumentar o tempo ocioso, acarretando em aumento na frequência de estereotipias (Johnson et al., 1998). Desta forma, deve-se ressaltar a importância da permanência dos animais em pastagem, a qual possibilita a busca e a ingestão de alimento de forma lenta e compatível com as características anatômicas e fisiológicas dos equinos (NRC, 2007).

Os potros dos tratamentos confinados (T50C e T100C) consumiram forragem por mais tempo $(P<0,05)$ no período da tarde, em comparação com a manhã, o que pode estar relacionado ao condicionamento de manejo no qual a percepção do confinamento desencadeia tal comportamento.

A velocidade de colheita da forragem avaliada no período diurno pela taxa de bocados, apresentou variação entre os tratamentos, principalmente associada com o tempo de pastejo, demonstrando a importância desta variável no contexto do comportamento alimentar. $\mathrm{Na}$ Tabela 3 observa-se que os animais dos tratamentos confinados no período noturno apresentam maior $(P<0,05)$ velocidade de ingestão do que os que permaneceram todo tempo em pastagem. Esta maior taxa de bocados pode ser um indicativo da tentativa dos animais em compensar o tempo que esses estiveram sem acesso à pastagem, mesmo com outros tipos de alimento à disposição. O confinamento interferiu diretamente no comportamento ingestivo, pois os animais do tratamento T50C incrementaram a ingestão de alimento no período da tarde por aumento na velocidade de colheita da forragem, sem aumentar o tempo de pastejo (Tabela 3).

A caracterização do número de bocados por período permite refletir a respeito das estratégias utilizadas pelos 
Tabela 4. Peso e escore corporal dos potros do início ao final do experimento (110 dias)

\begin{tabular}{ccccccc}
\hline Tratamento & $\begin{array}{c}\text { Peso inicial } \\
(\mathrm{kg})\end{array}$ & $\begin{array}{c}\text { Peso final } \\
(\mathrm{kg})\end{array}$ & $\begin{array}{c}\text { Ganho no } \\
\text { período }(\mathrm{kg})\end{array}$ & $\begin{array}{c}\text { Ganho diário } \\
(\text { gramas })\end{array}$ & $\begin{array}{c}\text { Escore } \\
\text { inicial }\end{array}$ & $\begin{array}{c}\text { Escore } \\
\text { final }\end{array}$ \\
\hline T100 C & 196 & 242 & $46^{\mathrm{b}}$ & $0,42^{\mathrm{b}}$ & $2,8^{\mathrm{a}}$ & $3,1^{\mathrm{a}}$ \\
T50 C & 185 & 239 & $54^{\mathrm{a}, \mathrm{b}}$ & $0,49^{\mathrm{a}, \mathrm{b}}$ & $2,7^{\mathrm{a}}$ & $2,9^{\mathrm{a}}$ \\
TP & 180 & 247 & $67^{\mathrm{a}}$ & $0,61^{\mathrm{a}}$ & $2,8^{\mathrm{a}}$ & $3,2^{\mathrm{a}}$ \\
\hline
\end{tabular}

Letras minúsculas diferentes na coluna diferem entre si pelo teste de Duncan $(P<0,05)$.

equinos frente ao manejo estabelecido pelo homem. Almeida (2001) encontrou em experimento feito com éguas da raça Mangalarga Marchador valores médios de 23,5 bocados por minuto. Santos (2006) relatou valores variando de 21,22 até 26,76 bocados por minutos. A taxa de bocados é um mecanismo compensatório para manter a ingestão de matéria seca relativamente constante (Chacon et al., 1978; Newman, 1994). Isso explica a tentativa dos potros nos tratamentos T100C e T50C em manter a ingestão de alimento constante por meio do aumento da velocidade de ingestão em um dos períodos avaliados.

O peso e escore corporal dos potros utilizados apresentaram-se satisfatórios no período avaliado para os três tratamentos. Os animais do T100C apresentaram menores ganhos em massa corpórea em relação aos animais exclusivamente mantidos em pastagem (Tabela 4).

O alimento concentrado na dieta dos potros não promoveu melhores ganhos diários e no período avaliado. Os animais mantidos exclusivamente em pastagem (TP) apresentaram boa capacidade de colheita de nutrientes, em condições de massa de forragem disponível não limitante, refletida pelo desempenho. A pastagem é composta por estruturas distintas e de composição química variável nos estádios de desenvolvimento, resultando em dosséis heterogêneos (Singer et al., 1999). Os cavalos exploram esta heterogeneidade por meio da seletividade nas plantas individualmente, consumindo as de maior digestibilidade (Coleman, 1992).
O aporte nutricional é dependente da participação de cada estrutura no dossel forrageiro e, também, do tempo disponibilizado para os animais 0 explorarem (Dittrich, 2010).

\section{CONCLUSÃO}

O confinamento noturno de potros diminui o tempo de ingestão alimentar diária mesmo com feno a disposição em quantidades superiores às necessidades. Esses animais aumentam a velocidade de ingestão de pasto durante o dia.

A pastagem de hemártria, sem limitação de oferta, permite adequado crescimento de potros da raça Mangalarga Marchador.

\section{REFERÊNCIAS}

ALMEIDA, F.Q.; BRITO, C.O.; LANA, A.M.Q. et al. Ingestive behaviour in grazing mares. In: Equine Nutrition and Physiology Symposium, 17., Lexington, 2001. Proceedings... Lexington: ENPS, 2001. p.479-483.

CHACON E.A.; STOBBS, T.H.; DALE, M.B. Influence of sward characteristics on grazing behaviour and growth of Hereford steers grazing tropical grass pastures. Australian Journal of Agricultural Research, v.29, n.1 p.89-102, 1978.

COLEMAN, S.W. Plant-animal interface. Journal of Productive Agriculture, v.5, p.7-13, 1992.

DITTRICH, J.R. Relações entre a estrutura das pastagens e a seletividade de eqüinos em pastejo. 2001. Curitiba, $77 \mathrm{f}$. Tese (Doutorado em Agronomia) - Universidade Federal do Paraná.

DITTRICH, J.R.; CARVALHO, P.C.F.; MORAES, A. et al. Preferência de eqüinos em pastejo: efeito da altura de dosséis de gramíneas do 
gênero Cynodon. Archives of Veterinary

Science, v.10, n.2, p.61-67, 2005.

DITTRICH, J.R.; CARVALHO, P.C.F.;

DITTRICH, R.L. et al. Comportamento ingestivo de eqüinos em pastagens. Archives of

Veterinary Science, v.12, n.3. p.1-8, 2007.

DITTRICH, J.R.; MELO, H.A.; AFONSO, A.M.C.F. et al. Comportamento ingestivo de equinos e a relação com o aproveitamento das forragens e bem-estar dos animais. Revista Brasileira de Zootecnia, v.39, supl. esp., p.130137, 2010.

DUNCAN, P. Time-budgets of Camargue horses. II. Time-budgets of adult horses and weaned sub-adults. Behaviour, v.72, p.26-49, 1980.

ELLIS, A.D.; HILL, J. Nutritional Physiology of the Horse. Nottingham University Press, 2005. $361 p$.

HENNEKE, D.R.; POTTER, G.D.; KREIDER, J.L. et al. Relationship between condition score, physical measurements and body fat percentage in mares. Equine Veterinary Journal, n.4, v.15, p.371-372, 1983.

HODGSON, J. Ingestive behaviour. In: LEAVER, J.D. (Ed.) Herbage intake handbook. Hurley: The British Grassland Society. 1982. p.113-138.

JANIS, C. The evolutionary strategy of the Equidae and the origin of rumen and cecal digestion. Evolution, v.30, p.757-774, 1975.

JOHNSON, K.G.J., TYRRELL, J.; ROWE, B. et al. Behavior changes in stable horses given nontherapeutic levels of virginiamycin. Equine veterinary Journal, v.30, p.139-143, 1998.

MARTIN, P.; BATESON, P. Measuring behaviour: an introductory guide. Cambridge; Cambridge University Press, 1986. 200p.

MOTT, G.O.; LUCAS, H.L. The design, conduct and interpretation of grazing trials on cultivated and improved pastures. In: International Grassland Congress, 6., 1952, Pensylvania. Proceedings... Pensylvania: State College Press, 1952. p.1380-1385.
NATIONAL RESEARCH COUNCIL - NRC. Nutrients requirements of domestic horses. 6 ed. Washington, D.C.: National Academy of Scince, 2007. 341p.

NEWMAN, J.A.; PARSONS, A.J.; PENNING, P.D. A note on the behavioral strategies used by grazing animals to alter their intake rates. Grass and Forage Science, v.49, p.502-505, 1994.

POND, K.R.; DUGAN, D.K.; BARNEET, D.T. et al. Grazing behavior of mares and steers on orchardgrass and mares on common Bermuda grass. In: Equine Nutrition and Physiology Symposium, 14., Raleigh. Proceedings... Raleigh: North Carolina State University Press, 1993. p.236.

RALSTON, S.L.; BAILE, C.A. Plasma glucose and insulin concentration in feeding behavior in ponies. Journal Animal Science, v.54, n.6, p.1132-1137, 1982.

SANTOS, E.M.; ZANINE ,A.M.; PARENTE, H.N. et al. Comportamento ingestivo de eqüinos em pastagens de grama batatais (Paspalum notatum) e braquiarinha (Brachiaria decumbens) na região centro-oeste do Brasil. Ciência Rural, v.36, n.5, 2006.

SINGER, J.W.; BOBSIN, N.; BAMKA, W.J. et al. Horse pasture management. Journal of Equine Veterinary Science, v.19, n.9, p.540-592, 1999.

WINSKILL, L.C.; WARAN, N.K.; YOUNG, R.J. The effect of a foraging device (a modified 'Edinburgh Foodball') on the behaviour of the stabled horse. Applied Animal Behaviour Science, v.48, p.25-35, 1996.

ZANINE, A.M.; SANTOS, E.M.; PARENTE, H.N. et al. Diferenças entre sexos para as atividades de pastejo de eqüinos no nordeste do Brasil. Archivos de Zootecnia, v.55, n.210, p.139-147, 2006. 